\title{
Implicit Iterative Scheme for a Countable Family of Nonexpansive Mappings in 2-Uniformly Smooth Banach Spaces
}

\author{
Ming Tian and Xin Jin \\ College of Science, Civil Aviation University of China, Tianjin 300300, China \\ Correspondence should be addressed to Ming Tian; tianming1963@126.com
}

Received 1 January 2013; Accepted 22 March 2013

Academic Editor: Qamrul Hasan Ansari

Copyright (C) 2013 M. Tian and X. Jin. This is an open access article distributed under the Creative Commons Attribution License, which permits unrestricted use, distribution, and reproduction in any medium, provided the original work is properly cited.

Implicit Mann process and Halpern-type iteration have been extensively studied by many others. In this paper, in order to find a common fixed point of a countable family of nonexpansive mappings in the framework of Banach spaces, we propose a new implicit iterative algorithm related to a strongly accretive and Lipschitzian continuous operator $F: x_{n}=\alpha_{n} \gamma V\left(x_{n}\right)+\beta_{n} x_{n-1}+((1-$ $\left.\left.\beta_{n}\right) I-\alpha_{n} \mu F\right) T_{n} x_{n}$ and get strong convergence under some mild assumptions. Our results improve and extend the corresponding conclusions announced by many others.

\section{Introduction}

Let $X$ be a real $q$-uniformly smooth Banach space with induced norm $\|\cdot\|, q>1$. Let $X^{*}$ be the dual space of $X$. Let $J_{q}$ denote the generalized duality mapping from $X$ into $2^{X^{*}}$ given by $J_{q}(x)=\left\{f \in X^{*}:\langle x, f\rangle=\|x\|^{q},\|f\|=\|x\|^{q-1}\right.$, $x \in X$ \}. In our paper, we consider real 2-uniformly smooth Banach spaces, that is, $q=2$, so the normalized duality mapping is $J(x)=\left\{f \in X^{*}:\langle x, f\rangle=\|x\|^{2},\|f\|=\|x\|, x \in\right.$ $X$ \}. If $X$ is smooth, then $J$ is single valued. Throughout this paper, we use $\operatorname{Fix}(T)$ to denote the fixed points set of the mapping $T$.

In what follows, we write $x_{n} \rightarrow x$ to indicate that the sequence converges weakly to $x . x_{n} \rightarrow x$ implies that the sequence converges strongly to $x$.

Given a nonlinear operator $\Gamma: X \rightarrow X$, it is well-known that the generalized variational inequality problem $\operatorname{VIP}(\Gamma, X)$ over $X$ is to find a $x^{*} \in X$, such that

$$
\left\langle\Gamma x^{*}, j\left(x-x^{*}\right)\right\rangle \geq 0, \quad \forall x \in X .
$$

Scholars mainly proposed iterative algorithms to solve the generalized variational inequalities, and some of them focused on the existence of the solutions of generalized variational inequalities; see $[1,2]$ and references therein.
Variational inequalities are developed from operator equations and have been playing an essential role in management science, mechanics, and finance. As for mathematics, variational inequality problems mainly originate from partial differential equations, optimization problems; see [3-7] and references therein.

Definition 1. A mapping T is said to be

(1) $\eta$-strongly accretive if for each $x, y \in X$, there exists a $j(x-y) \in J(x-y)$ and $\eta>0$, such that

$$
\langle T x-T y, j(x-y)\rangle \geq \eta\|x-y\|^{q} ;
$$

(2) $L$-Lipschitzian continuous if for each $x, y \in X$, there exists a constant $L>0$, such that

$$
\|T x-T y\| \leq L\|x-y\| .
$$

In particular, $T$ is called nonexpansive if $L=1$; it is said to be contractive if $L<1$.

Yamada [3] introduced the hybrid steepest descent method:

$$
x_{n+1}=\left(I-\mu \lambda_{n} F\right) T x_{n}, \quad \forall n \geq 0,
$$


where $T$ is a nonexpansive mapping in Hilbert spaces. Under some appropriate conditions, Yamada [3] proved that the sequence $\left\{x_{n}\right\}$ generated by (4) converges strongly to the unique solution $x^{*} \in \operatorname{Fix}(T)$ of the variational inequality: $\operatorname{VIP}(F, X):\left\langle F x^{*}, x-x^{*}\right\rangle \geq 0$, for all $x \in \operatorname{Fix}(T)$.

Moudafi [4] introduced the classical viscosity approximation method for nonexpansive mappings and defined a sequence $\left\{x_{n}\right\}$ by

$$
x_{n+1}=\alpha_{n} f\left(x_{n}\right)+\left(1-\alpha_{n}\right) T x_{n}, \quad \forall n \geq 0,
$$

where $\left\{\alpha_{n}\right\}$ is a sequence in $(0,1) . \mathrm{Xu}[6]$ proved that under certain appropriate conditions on $\left\{\alpha_{n}\right\}$, the sequence $\left\{x_{n}\right\}$ generated by (5) converges strongly to the unique solution $x^{*} \in C$ of the variational inequality: $\left\langle(I-f) x^{*}, x-x^{*}\right\rangle \geq 0$, for all $x \in \operatorname{Fix}(T)$, (where $C=\operatorname{Fix}(T))$ in Hilbert spaces as well as in some Banach spaces.

Marino and $\mathrm{Xu}$ [7] considered the following general iterative method in Hilbert spaces:

$$
x_{n+1}=\alpha_{n} \gamma f\left(x_{n}\right)+\left(I-\alpha_{n} A\right) T x_{n}, \quad \forall n \geq 0,
$$

where $A$ is a strongly positive bounded linear operator. It is proved that if the sequence $\left\{\alpha_{n}\right\}$ satisfies appropriate conditions, the sequence $\left\{x_{n}\right\}$ generated by (6) converges strongly to the unique solution $\widetilde{x} \in C$ of the variational inequality: $\langle(\gamma f-A) \tilde{x}, x-\tilde{x}\rangle \leq 0$, for all $x \in C$, where $C$ is the fixed points set of a nonexpansive mapping $T$.

Tian [8] considered the following general iterative algorithm (GIA) in Hilbert spaces:

$$
x_{n+1}=\alpha_{n} \gamma f\left(x_{n}\right)+\left(I-\mu \alpha_{n} F\right) T x_{n}, \quad \forall n \geq 0 .
$$

It is proved that if the sequence $\left\{\alpha_{n}\right\}$ satisfies appropriate conditions, the sequence $\left\{x_{n}\right\}$ generated by (7) converges strongly to the unique solution $\tilde{x} \in \operatorname{Fix}(T)$ of the variational inequality: $\langle(\gamma f-\mu F) \tilde{x}, x-\tilde{x}\rangle \leq 0$, for all $x \in \operatorname{Fix}(T)$.

In 2001, Soltuz [9] introduced the following backward Mann scheme iteration:

$$
x_{n}=\alpha_{n} x_{n-1}+\left(1-\alpha_{n}\right) T x_{n}, \quad \forall n \geq 1,
$$

where $T$ is a nonexpansive mapping and got strong convergence in Hilbert spaces.

In order to find a common fixed point of a finite family of nonexpansive mappings $\left\{T_{i}: i \in J\right\}$, where $J$ stands for $\{1,2,3, \ldots, N\}$, in 2001, Xu and Ori [10] introduced the following implicit process:

$$
x_{n}=\alpha_{n} x_{n-1}+\left(1-\alpha_{n}\right) T_{n} x_{n}, \quad \forall n \geq 1,
$$

where $T_{n}=T_{n(\bmod N)}$, and a weak convergence is obtained in real Hilbert spaces.

Ceng et al. [11] introduced an iterative algorithm to find a common fixed point of a finite family of nonexpansive semigroups in reflexive Banach spaces with a weak sequentially continuous duality mapping, which satisfy the uniformly asymptotical regularity condition:

$$
\begin{gathered}
x_{n+1}=\left(1-\alpha_{n}-\beta_{n}\right) x_{n}+\alpha_{n} f\left(y_{n}\right)+\beta_{n} T_{r_{n}} y_{n}, \\
y_{n}=\left(1-\gamma_{n}\right) x_{n}+\gamma_{n} T_{r_{n}} x_{n} .
\end{gathered}
$$

Under some appropriate conditions one the parameter sequences $\left\{\alpha_{n}\right\},\left\{\beta_{n}\right\},\left\{\gamma_{n}\right\}$, and the sequence $\left\{x_{n}\right\}$ generated by (10) converges strongly to the approximate solution of a variational inequality problem.

In order to find a common element of the solution set of a general system of variational inequalities and the fixedpoint set of the mapping $S$, Ceng et al. [12] constructed a new relaxed extragradient iterative method:

$$
\begin{gathered}
u_{n}=P_{C}\left[P_{C}\left(x_{n}-\mu_{2} B_{2} x_{n}\right)-\mu_{1} B_{1} P_{C}\left(x_{n}-\mu_{2} B_{2} x_{n}\right)\right], \\
z_{n}=P_{C}\left(u_{n}-\lambda_{n} A u_{n}\right), \\
y_{n}=\alpha_{n} f\left(x_{n}\right)+\left(1-\alpha_{n}\right) P_{C}\left(u_{n}-\lambda_{n} A z_{n}\right), \\
x_{n+1}=\beta_{n} x_{n}+\gamma_{n} y_{n}+\delta_{n} S y_{n}, \quad \forall n \geq 0 .
\end{gathered}
$$

Under mild assumptions, they obtained a strong convergence theorem.

Yao et al. [13] introduced the following Halpern-type implicit iterative method where $T$ is a continuous pseudocontraction:

$$
x_{n}=\alpha_{n} u+\beta_{n} x_{n-1}+\gamma_{n} T x_{n}, \quad \forall n \geq 1
$$

and obtained a strong convergence theorem in Banach spaces.

$\mathrm{Hu}$ [14] introduced an iteration for a nonexpansive mapping in Banach spaces, which guarantee a uniformly Gêteaux differentiable norm as follows:

$$
x_{n+1}=\alpha_{n} u+\beta_{n} x_{n}+\gamma_{n} T x_{n}, \quad \forall n \geq 0,
$$

and several strong convergent theorems are obtained.

Very recently, Jung [15] proposed an iterative process in the frame of Hilbert spaces as follows:

$$
\begin{aligned}
x_{n+1}= & \alpha_{n} \gamma f\left(x_{n}\right)+\beta_{n} x_{n} \\
& +\left(\left(1-\beta_{n}\right) I-\alpha_{n} \mu F\right) P_{C} S x_{n}, \quad \forall n \geq 0,
\end{aligned}
$$

where $S$ is a mapping defined by $S x=k x+(1-k) T x$ and $T$ is a $k$-strictly pseudocontraction. Strong convergence theorems are established.

Motivated and inspired by Soltuz [9], Xu and Ori [10], Ceng et al. [11], Ceng et al. [12], Yao et al. [13], Hu [14], and Jung [15], we consider the following new implicit iteration in real 2-uniformly smooth Banach spaces:

$$
\begin{aligned}
x_{n}= & \alpha_{n} \gamma V\left(x_{n}\right)+\beta_{n} x_{n-1} \\
& +\left(\left(1-\beta_{n}\right) I-\alpha_{n} \mu F\right) T_{n} x_{n}, \quad \forall n \geq 1,
\end{aligned}
$$

where $\left\{\alpha_{n}\right\}$ and $\left\{\beta_{n}\right\}$ are real sequences in $(0,1), V$ is an $L$ Lipschitzian continuous with Lipschitzian constant $L>0$, $F$ is an $\eta$-strongly accretive and $\kappa$-Lipschitzian continuous mapping with $\kappa>0$ and $\eta>0$, and $\left\{T_{i}\right\}_{i=1}^{\infty}$ is a countable family of nonexpansive mappings.

In this paper, we prove that the implicit iterative process (15) has strong convergence and find the unique solution $\tilde{x}$ of variational inequality:

$$
\langle(\mu F-\gamma V) \tilde{x}, j(x-\tilde{x})\rangle \geq 0, \quad \forall x \in S=\bigcap_{n=1}^{\infty} \operatorname{Fix}\left(T_{n}\right) \neq \emptyset .
$$


Our results improve and extend the corresponding conclusions announced by many others.

\section{Preliminaries}

Let $S_{X}=\{x \in X:\|x\|=1\}$. Then the norm of $X$ is said to be Gâteaux differentiable if

$$
\Delta=\lim _{t \rightarrow 0} \frac{\|x+t y\|-\|x\|}{t}
$$

exists for each $x, y \in S_{X}$. In this case, $X$ is said to be smooth. The norm of $X$ is called uniformly Gâteaux differentiable, if for each $y \in S_{X}, \Delta$ is attained uniformly for $x \in S_{X}$. The norm of $X$ is called Fréchet differentiable, if for each $x \in S_{X}, \Delta$ is attained uniformly for $y \in S_{X}$. The norm of $X$ is called uniformly Fréchet differentiable, if $\Delta$ is attained uniformly for $x, y \in S_{X}$. It is well known that (uniformly) Fréchet differentiability of the norm of $X$ implies (uniformly) Gâteaux differentiability of the norm of $X$. If the norm on $X$ is uniformly Gâteaux differentiable, the generalized duality mapping $J_{q}$ is single-valued and strong-weak ${ }^{*}$ uniformly continuous on any bounded subsets of $X$.

Let $\rho_{X}:[0, \infty) \rightarrow \infty$ be the modulus of smoothness of $X$ defined by

$$
\rho_{X}(t)=\sup \left\{\frac{1}{2}(\|x+y\|+\|x-y\|)-1: x \in S_{X},\|y\| \leq t\right\} .
$$

A Banach space $X$ is said to be uniformly smooth if $\rho_{X}(t) / t \rightarrow 0$ as $t \rightarrow 0$. A Banach space is said to be $q$-uniformly smooth, if there exists a fixed constant $c>0$, such that $\rho_{X}(t) \leq c t^{q}$. It is well known that the $X$ is uniformly smooth if and only if the norm of $X$ is uniformly Fréchet differentiable [16].

The so-called gauge function $\varphi$ is defined as follows: let $\varphi:[0, \infty):=\mathbb{R}^{+} \rightarrow \mathbb{R}^{+}$be a continuous strictly increasing function, such that $\varphi(0)=0$ and $\varphi(t) \rightarrow \infty$ as $t \rightarrow \infty$. The duality mapping $J_{\varphi}: X \rightarrow 2^{X^{*}}$ associated with a gauge function $\varphi$ is defined by

$$
\begin{aligned}
& J_{\varphi}(x) \\
& \quad=\left\{f \in X^{*}:\langle x, f\rangle=\|x\| \varphi(\|x\|),\|f\|=\varphi(\|x\|), x \in X\right\} .
\end{aligned}
$$

It is known that real 2-uniformly smooth Banach spaces have a weakly continuous duality mapping with a guage function $\varphi(t)=t$, which is the same as the normalized duality mapping $J$. Set $\Phi(t)=\int_{0}^{t} \varphi(\tau) d \tau$, for all $t \geq 0$, then $J_{\varphi}(x)=\partial \Phi(\|x\|)$, where $\partial$ denotes the subdifferential in the sense of convex analysis. In fact, for $0 \leq k \leq 1$, we have $\varphi(k t) \leq \varphi(t)$ and

$$
\begin{aligned}
\Phi(k t) & =\int_{0}^{k t} \varphi(\tau) d \tau=k \int_{0}^{t} \varphi(k \tau) d \tau \\
& \leq k \int_{0}^{t} \varphi(\tau) d \tau=k \Phi(t) .
\end{aligned}
$$

Lemma 2 (see [17]). Let $X$ be a real q-uniformly smooth Banach space for some $q>1$, then there exists some positive constant $d_{q}$, such that

$$
\begin{array}{r}
\|x+y\|^{q} \leq\|x\|^{q}+q\left\langle y, j_{q}(x)\right\rangle+d_{q}\|y\|^{q}, \\
\forall x, y \in X, j_{q}(x) \in J_{q}(x),
\end{array}
$$

in particular, if $X$ is a real 2-uniformly smooth Banach space, then there exists a best smooth constant $K>0$, such that

$$
\begin{array}{r}
\|x+y\|^{2} \leq\|x\|^{2}+2\langle y, j(x)\rangle+2\|K y\|^{2} \\
\forall x, y \in X, j_{q}(x) \in J_{q}(x) .
\end{array}
$$

Lemma 3 (see [18]). Assume that a Banach space $X$ has a weakly continuous duality mapping $J_{q}$ :

(i) for all $x, y \in X$, the following inequality holds:

$$
\Phi(\|x+y\|) \leq \Phi(\|x\|)+\left\langle y, j_{q}(x+y)\right\rangle
$$

in particular, for all $x, y \in X$, there holds:

$$
\|x+y\|^{2} \leq\|x\|^{2}+2\langle y, j(x+y)\rangle
$$

(ii) assume that a sequence $\left\{x_{n}\right\} \subset X$ converges weakly to a point $x \in X$, then the following equation holds:

$$
\begin{aligned}
\limsup _{n \rightarrow \infty} \Phi\left(\left\|x_{n}-y\right\|\right)= & \limsup _{n \rightarrow \infty} \Phi\left(\left\|x_{n}-x\right\|\right) \\
& +\Phi(\|y-x\|), \quad \forall x, y \in X .
\end{aligned}
$$

Lemma 4 (see [19]). Assume that $\left\{a_{n}\right\}$ is a sequence of nonnegative real numbers, such that

$$
a_{n+1} \leq\left(1-\gamma_{n}\right) a_{n}+\gamma_{n} \delta_{n}, \quad \forall n \geq 0,
$$

where $\left\{\gamma_{n}\right\}$ is a sequence in $(0,1)$ and $\left\{\delta_{n}\right\}$ is a sequence in $\mathbb{R}$, such that

(a) $\sum_{n=0}^{\infty} \gamma_{n}=\infty$;

(b) $\lim \sup _{n \rightarrow \infty} \delta_{n} \leq 0$ or $\sum_{n=0}^{\infty}\left|\gamma_{n} \delta_{n}\right|<\infty$.

Then $\lim _{n \rightarrow \infty} a_{n}=0$.

Lemma 5. Let $X$ be a real 2-uniformly smooth Banach space. Let $T$ be a nonexpansive mapping over $X$, and let $F: X \rightarrow$ $X$ be an $\eta$-strongly accretive and $\kappa$-Lipschitzian continuous mapping with $\kappa>0$ and $\eta>0$. For $0<t<\sigma \leq 1$ and $\mu \in\left(0, \min \left\{1, \eta / K^{2} \kappa^{2}\right\}\right)$, set $\tau=\mu\left(\eta-\mu K^{2} \kappa^{2}\right)$, and define a mapping $T^{t}: X \rightarrow X$ by $T^{t}:=\sigma I-t \mu F$. Then $T^{t}$ is a contraction on $X$; that is, $\left\|T^{t} x-T^{t} y\right\| \leq(\sigma-t \tau)\|x-y\|$. 
Proof. From $0<\mu<\eta / K^{2} \kappa^{2}$, we have $\eta-\mu K^{2} \kappa^{2}>0$. Setting $\eta<1 / 2$, we have $0<2\left(\eta-\mu K^{2} \kappa^{2}\right)<1$. For each $x, y \in X$, by Lemma 2, we have

$$
\begin{aligned}
\| T^{t} x- & T^{t} y \|^{2} \\
= & \|\sigma(x-y)-t \mu(F x-F y)\|^{2} \\
\leq & \sigma^{2}\|x-y\|^{2}-\sigma 2 t \mu\langle F x-F y, j(x-y)\rangle \\
& +2 K^{2} t^{2} \mu^{2}\|F x-F y\|^{2} \\
\leq & \sigma^{2}\|x-y\|^{2}-2 \sigma t \mu \eta\|x-y\|^{2} \\
& +2 K^{2} t^{2} \mu^{2} \kappa^{2}\|x-y\|^{2} \\
\leq & {\left[\sigma^{2}-2 \sigma t \mu\left(\eta-\mu K^{2} \kappa^{2}\right)\right]\|x-y\|^{2} } \\
= & \left(\sigma^{2}-2 \sigma t \tau\right)\|x-y\|^{2} \\
\leq & (\sigma-t \tau)^{2}\|x-y\|^{2} .
\end{aligned}
$$

Hence, it implies that

$$
\left\|T^{t} x-T^{t} y\right\| \leq(\sigma-t \tau)\|x-y\| .
$$

This completes the proof.

To deal with a family of mappings, we will introduce the following concept called the AKTT condition.

Definition 6 (see [20]). Let $X$ be a real Banach space, let $C$ be a nonempty subset of $X$, and let $\left\{T_{n}\right\}_{n=1}^{\infty}$ be a countable family of mappings of $C$ with $\bigcap_{n=1}^{\infty} \operatorname{Fix}\left(T_{n}\right) \neq \emptyset$. Then $\left\{T_{n}\right\}$ is said to satisfy the AKTT condition, if for any bounded subset $D$ of $C$, the following inequality holds:

$$
\sum_{n=1}^{\infty} \sup \left\{\left\|T_{n+1} x-T_{n} x\right\|: x \in D\right\}<\infty .
$$

Lemma 7 (see [20]). Let $X$ be a Banach space, let $C$ be a nonempty closed subset of $X$, and let $\left\{T_{n}\right\}$ be a family of selfmappings of $C$ satisfying the AKTT condition. Then for each $x \in C,\left\{T_{n} x\right\}$ converges strongly to a point in $C$. Moreover, let the mapping $T$ be defined by

$$
T x=\lim T_{n} x, \quad \forall x \in C .
$$

Then for any bounded subset $D$ of $C$, the following equality holds:

$$
\limsup _{n \rightarrow \infty} \sup \left\{\left\|T x-T_{n} x\right\|: x \in D\right\}=0 \text {. }
$$

Lemma 8 (see [1]). Suppose that $q>1$. Then the following inequality holds:

$$
a b \leq \frac{1}{q} a^{q}+\frac{q-1}{q} b^{q /(q-1)},
$$

for arbitrary positive real numbers $a$ and $b$.

\section{Main Results}

In order to obtain the main results, we divide this section into 3 parts. In Proposition 9, we give the path convergence. In Proposition 10, under the demiclosed assumption and combined with Proposition 9, we find the unique solution of a variational inequality. In Theorem 11, we prove that the sequence $\left\{x_{n}\right\}$ defined by the implicit scheme (15) converges strongly to the unique solution of (16).

Throughout this paper, we assume that $X$ is a real 2uniformly smooth Banach space, which guarantees a weakly continuous duality $J$ as proposed in Section 1.

Proposition 9 (the path convergence). Let $T: X \rightarrow X$ be a nonexpansive mapping with $\operatorname{Fix}(T) \neq \emptyset$, and let $V: X \rightarrow$ $X$ be an L-Lipschitzian continuous mapping with Lipschitzian constant $L>0 . F: X \rightarrow X$ is an $\eta$-strongly accretive and $\kappa$ Lipschitzian continuous mapping with $\kappa>0$ and $\eta>0$. For $\epsilon$ $(0,1)$, let $\mu \in\left(0, \min \left\{1, \eta / K^{2} \kappa^{2}\right\}\right)$, and set $\tau=\mu\left(\eta-\mu K^{2} \kappa^{2}\right)$ and $0<\gamma<\tau / L$. Then assume that $\left\{x_{t}\right\}$ is defined by

$$
x_{t}=t \gamma V\left(x_{t}\right)+(I-t \mu F) T x_{t} .
$$

Then $\left\{x_{t}\right\}$ converges strongly as $\rightarrow 0^{+}$to a fixed point $\tilde{x}$ of $T$, which is the unique solution of the variational inequality VIP:

$$
\langle(\mu F-\gamma V) \tilde{x}, j(x-\tilde{x})\rangle \geq 0, \quad \forall x \in \operatorname{Fix}(T) .
$$

Proof. Consider a mapping $S_{t}$ on $X$ defined by

$$
S_{t} x=t \gamma V\left(x_{t}\right)+(I-t \mu F) T x_{t}, \quad \forall x \in X .
$$

It is easy to see that $S_{t}$ is a contraction. Indeed, for any $x, y \in$ $X$, by Lemma 5 , we have

$$
\begin{aligned}
\left\|S_{t} x-S_{t} y\right\| \leq & t \gamma\|V(x)-V(y)\| \\
& +\|(I-t \mu F) T x-(I-t \mu F) T y\| \\
\leq & {[1-t(\tau-\gamma L)]\|x-y\| . }
\end{aligned}
$$

Hence, by the Banach contraction mapping principle, $S_{t}$ has a unique fixed point, denoted by $x_{t}$, which uniquely solves the fixed point equation (33).

We divided the proof into several steps.

Step 1. We show the uniqueness of the solution of the variational inequality (34). Assume that both $x_{1} \in \operatorname{Fix}(T)$ and $x_{2} \in \operatorname{Fix}(T)$ are solutions of the variational inequality (34), then we have

$$
\begin{aligned}
& \left\langle(\mu F-\gamma V) x_{1}, j_{q}\left(x_{2}-x_{1}\right)\right\rangle \geq 0, \\
& \left\langle(\mu F-\gamma V) x_{2}, j\left(x_{1}-x_{2}\right)\right\rangle \geq 0 .
\end{aligned}
$$

Adding up (37) yields

$$
\left\langle(\mu F-\gamma V) x_{1}-(\mu F-\gamma V) x_{2}, j\left(x_{2}-x_{1}\right)\right\rangle \geq 0 .
$$


Indeed, from the given conditions $\mu \in\left(0, \min \left\{1, \eta / K^{2} \kappa^{2}\right\}\right)$, and $\tau=\mu\left(\eta-\mu K^{2} \kappa^{2}\right), 0<\gamma<\tau / L$, we have

$$
\begin{aligned}
\langle(\mu F- & \left.\gamma V) x_{1}-(\mu F-\gamma V) x_{2}, j\left(x_{1}-x_{2}\right)\right\rangle \\
= & \mu\left\langle F x_{1}-F x_{2}, j\left(x_{1}-x_{2}\right)\right\rangle \\
& -\gamma\left\langle V x_{1}-V x_{2}, j\left(x_{1}-x_{2}\right)\right\rangle \\
\geq & \mu \eta\left\|x_{1}-x_{2}\right\|^{2}-\gamma L\left\|x_{1}-x_{2}\right\|^{2} \\
= & (\mu \eta-\gamma L)\left\|x_{1}-x_{2}\right\|^{2} \geq 0 .
\end{aligned}
$$

Thus, we conclude that $x_{1}=x_{2}$. So the uniqueness of the variational inequality (35) is guaranteed.

Step 2. We show that $\left\{x_{t}\right\}$ is bounded. Taking $p \in \operatorname{Fix}(T)$, it follows from Lemma 5 that

$$
\begin{aligned}
\| x_{t}- & p \|^{2} \\
= & \left\langle t \gamma V\left(x_{t}\right)+(I-t \mu F) T x_{t}-p, j\left(x_{t}-p\right)\right\rangle \\
= & t\left\langle\gamma V\left(x_{t}\right)-\mu F p, j\left(x_{t}-p\right)\right\rangle \\
& +\left\langle(I-t \mu F) T x_{t}-(I-t \mu F) p, j\left(x_{t}-p\right)\right\rangle \\
\leq & t\left\langle\gamma V\left(x_{t}\right)-\gamma V(p)+\gamma V(p)-\mu F p, j\left(x_{t}-p\right)\right\rangle \\
& +\left\|(I-t \mu F) T x_{t}-(I-t \mu F) p\right\|\left\|x_{t}-p\right\| \\
\leq & t \gamma L\left\|x_{t}-p\right\|^{2}+t\|\gamma V(p)-\mu F p\|\left\|x_{t}-p\right\| \\
& +(1-t \tau)\left\|x_{t}-p\right\|^{2} \\
\leq & (1-t(\tau-\gamma L))\left\|x_{t}-p\right\|^{2} \\
& +t\|\gamma V(p)-\mu F p\|\left\|x_{t}-p\right\| .
\end{aligned}
$$

It follows that

$$
\left\|x_{t}-p\right\| \leq \frac{1}{\tau-\gamma L}\|\gamma V(p)-\mu F p\| .
$$

Hence $\left\{x_{t}\right\}$ is bounded, so are $\left\{V\left(x_{t}\right)\right\}$ and $\left.\left\{F T x_{t}\right)\right\}$.

Step 3. Next, we will show that $\left\{x_{t}\right\}$ has a subsequence converging strongly to $x^{*} \in \operatorname{Fix}(T)$.

Assume $t_{n} \rightarrow 0$, and set $x_{n}:=x_{t_{n}}$. By the definition of $\left\{x_{n}\right\}$, we have

$$
\left\|x_{n}-T x_{n}\right\|=t_{n}\left\|\gamma V\left(x_{n}\right)-\mu F T x_{n}\right\| \longrightarrow 0 .
$$

Since $\left\{x_{n}\right\}$ is bounded, there exists a subsequence $\left\{x_{n_{k}}\right\}$ of $\left\{x_{n}\right\}$ converging weakly to $x^{*} \in X$ as $k \rightarrow \infty$.

Set $x_{n}:=x_{n_{k}}$. Define a mapping $B: X \rightarrow \mathbb{R}$ by

$$
B(x)=\limsup _{n \rightarrow \infty} \Phi\left(\left\|x_{n}-x\right\|\right), \quad \forall x \in X .
$$

Again, $J$ is weakly continuous, by Lemma 3, and it follows that

$$
B(y)=B(x)+\Phi\left(\left\|y-x^{*}\right\|\right), \quad \forall y \in X .
$$

From (42), we have

$$
\begin{aligned}
B\left(T x^{*}\right) & =\limsup _{n \rightarrow \infty} \Phi\left(\left\|x_{n}-T x^{*}\right\|\right) \\
& =\limsup _{n \rightarrow \infty} \Phi\left(\left\|T x_{n}-T x^{*}+x_{n}-T x_{n}\right\|\right) \\
& =\limsup _{n \rightarrow \infty} \Phi\left(\left\|T x_{n}-T x^{*}\right\|\right) \\
& \leq \limsup _{n \rightarrow \infty} \Phi\left(\left\|x_{n}-x^{*}\right\|\right)=B\left(x^{*}\right),
\end{aligned}
$$

and we also note that

$$
\begin{aligned}
B\left(T x^{*}\right) & =\limsup _{n \rightarrow \infty} \Phi\left(\left\|x_{n}-x^{*}\right\|\right)+\Phi\left(\left\|T x^{*}-x^{*}\right\|\right) \\
& =B\left(x^{*}\right)+\Phi\left(\left\|T x^{*}-x^{*}\right\|\right),
\end{aligned}
$$

so, we obtain

$$
\Phi\left(\left\|T x^{*}-x^{*}\right\|\right) \leq 0 .
$$

This implies that $T x^{*}=x^{*}$; that is, $x^{*} \in \operatorname{Fix}(T)$.

By Lemma 5, we have

$$
\begin{aligned}
\| x_{n}- & x^{*} \|^{2} \\
= & \left\|t_{n}\left(\gamma V\left(x_{n}\right)-\mu F x^{*}\right)+\left(I-t_{n} \mu F\right) T x_{n}-\left(I-t_{n} \mu F\right) x^{*}\right\|^{2} \\
= & \left\langle\left(I-t_{n} \mu F\right) T x_{n}-\left(I-t_{n} \mu F\right) x^{*}, j\left(x_{n}-x^{*}\right)\right\rangle \\
& +t_{n}\left\langle\gamma V\left(x_{n}\right)-\mu F x^{*}, j\left(x_{n}-x^{*}\right)\right\rangle \\
\leq & \left(1-t_{n} \tau\right)\left\|x_{n}-x^{*}\right\|^{2} \\
& +t_{n}\left\langle\gamma V\left(x_{n}\right)-\gamma V\left(x^{*}\right), j\left(x_{n}-x^{*}\right)\right\rangle \\
& +t_{n}\left\langle\gamma V\left(x^{*}\right)-\mu F x^{*}, j\left(x_{n}-x^{*}\right)\right\rangle \\
\leq & \left(1-t_{n} \tau\right)\left\|x_{n}-x^{*}\right\|^{2}+t_{n} \gamma L\left\|x_{n}-x^{*}\right\|^{2} \\
& +t_{n}\left\langle\gamma V\left(x^{*}\right)-\mu F x^{*}, j\left(x_{n}-x^{*}\right)\right\rangle \\
= & \left(1-t_{n}(\tau-\gamma L)\right)\left\|x_{n}-x^{*}\right\|^{2} \\
& +t_{n}\left\langle\gamma V\left(x^{*}\right)-\mu F x^{*}, j\left(x_{n}-x^{*}\right)\right\rangle .
\end{aligned}
$$

This implies that

$$
\left\|x_{n}-x^{*}\right\|^{2} \leq \frac{1}{\tau-\gamma L}\left\langle\gamma V\left(x^{*}\right)-\mu F x^{*}, j\left(x_{n}-x^{*}\right)\right\rangle .
$$

Since $\left\{x_{n}\right\}$ is bounded, there exists a subsequence $\left\{x_{n_{k}}\right\}$ of $\left\{x_{n}\right\}$ satisfying

$$
\left\|x_{n_{k}}-x^{*}\right\|^{2} \leq \frac{1}{\tau-\gamma L}\left\langle\gamma V\left(x^{*}\right)-\mu F x^{*}, j\left(x_{n_{k}}-x^{*}\right)\right\rangle .
$$

Since the mapping $J$ is single-valued and weakly continuous, it follows from (50) that $\left\|x_{n_{k}}-x^{*}\right\|^{2} \rightarrow 0$ as $k \rightarrow \infty$. Thus, there exists a subsequence, such that $x_{n_{k}} \rightarrow x^{*}$. 
Step 4. Finally, we show that $x^{*}$ is the unique solution of variational inequality (34).

Since $x_{t}=t \gamma V\left(x_{t}\right)+(I-t \mu F) T x_{t}$, we can derive that

$$
(\mu F-\gamma V) x_{t}=-\frac{1}{t}(I-T) x_{t}+\mu\left(F x_{t}-F T x_{t}\right) .
$$

It follows that, for any $x \in \operatorname{Fix}(T)$,

$$
\begin{aligned}
\left\langle(\mu F-\gamma V) x_{t}, j\left(x_{t}-x\right)\right\rangle & \\
= & -\frac{1}{t}\left\langle(I-T) x_{t}-(I-T) x, j\left(x_{t}-x\right)\right\rangle \\
& +\mu\left\langle F x_{t}-F T x_{t}, j\left(x_{t}-x\right)\right\rangle .
\end{aligned}
$$

Since $T$ is a nonexpansive mapping, for all $x, y \in X$, we conclude that

$$
\begin{aligned}
& \langle(I-T) x-(I-T) y, j(x-y)\rangle \\
& \quad=\langle x-y, j(x-y)\rangle-\langle T x-T y, j(x-y)\rangle \\
& \quad \geq\|x-y\|^{2}-\|x-y\|^{2}=0 .
\end{aligned}
$$

Now replacing $t$ in (52) with $t_{n}$ and letting $n \rightarrow \infty$, from (42), we have that $F x_{t_{n}}-F T x_{t_{n}} \rightarrow 0$, thus we can conclude that

$$
\left\langle(\mu F-\gamma V) x^{*}, j\left(x^{*}-x\right)\right\rangle \leq 0, \quad \forall x \in \operatorname{Fix}(T) .
$$

So, $x^{*}$ is a solution of (34). Hence, $x^{*}=\tilde{x}$ by uniqueness. Therefore, $x_{t} \rightarrow \tilde{x}$ as $t \rightarrow 0^{+}$. This completes the proof.

Proposition 10 (the demiclosed result). Let $T: X \rightarrow X$ be a nonexpansive mapping with $\operatorname{Fix}(T) \neq \emptyset$, and let $V$ be an $L$ Lipschitzian continuous self-mapping on $X$ with Lipschitzian constant $L>0 . F: X \rightarrow X$ is an $\eta$-strongly accretive and $\kappa$-Lipschitzian continuous mapping with $\kappa>0$ and $\eta>0$. Assume that the net $\left\{x_{t}\right\}$ is defined as Proposition 9 which converges strongly as $t \rightarrow 0^{+}$to $\tilde{x} \in \operatorname{Fix}(T)$. Suppose that the sequence $\left\{x_{n}\right\} \subset X$ is bounded and satisfies the condition $\lim _{n \rightarrow \infty}\left\|x_{n}-T x_{n}\right\|=0$ (the so-called demiclosed property). Then the following inequality VIP holds:

$$
\limsup _{n \rightarrow \infty}\left\langle(\gamma V-\mu F) \tilde{x}, j\left(x_{n}-\tilde{x}\right)\right\rangle \leq 0 .
$$

Proof. Set $a_{n}(t)=\left\|T x_{n}-x_{n}\right\|\left\|x_{t}-x_{n}\right\|$. From the given condition and the boundness of $\left\{x_{t}\right\}$ and $\left\{x_{n}\right\}$, it is obvious that $a_{n}(t) \rightarrow 0$ when $n \rightarrow \infty$.

From (33) and the fact that $T$ is a nonexpansive mapping, we obtain that

$$
\begin{aligned}
\left\|x_{t}-x_{n}\right\|^{2} & \\
= & \left\langle\left(t \gamma V x_{t}+(I-t \mu F) T x_{t}\right)-x_{n}, j\left(x_{t}-x_{n}\right)\right\rangle \\
= & \left\langle T x_{t}-T x_{n}+T x_{n}-x_{n}+t\left(\gamma V x_{t}-\mu F x_{t}\right)\right. \\
& \left.+t\left(\mu F x_{t}-\mu F T x_{t}\right), j\left(x_{t}-x_{n}\right)\right\rangle
\end{aligned}
$$

$$
\begin{aligned}
\leq & \left\|x_{t}-x_{n}\right\|^{2}+\left\langle T x_{n}-x_{n}, j\left(x_{t}-x_{n}\right)\right\rangle \\
& +t\left\langle\gamma V x_{t}-\mu F x_{t}, j\left(x_{t}-x_{n}\right)\right\rangle \\
& +t\left\langle\mu F x_{t}-\mu F T x_{t}, j\left(x_{t}-x_{n}\right)\right\rangle \\
\leq & \left\|x_{t}-x_{n}\right\|^{2}+\left\|T x_{n}-x_{n}\right\|\left\|x_{t}-x_{n}\right\| \\
& +t\left\langle\gamma V x_{t}-\mu F x_{t}, j\left(x_{t}-x_{n}\right)\right\rangle \\
& +t \mu\left\|F x_{t}-F T x_{t}\right\|\left\|x_{t}-x_{n}\right\|,
\end{aligned}
$$

which implies that

$$
\begin{aligned}
\left\langle(\mu F-\gamma V) x_{t}, j\left(x_{t}-x_{n}\right)\right\rangle \leq & \frac{1}{t}\left\|T x_{n}-x_{n}\right\|\left\|x_{t}-x_{n}\right\| \\
& +\mu\left\|F x_{t}-F T x_{t}\right\|\left\|x_{t}-x_{n}\right\| .
\end{aligned}
$$

It follows that

$$
\begin{aligned}
& \limsup _{n \rightarrow \infty}\left\langle(\mu F-\gamma V) x_{t}, j\left(x_{t}-x_{n}\right)\right\rangle \\
& \quad \leq \mu\left\|F x_{t}-F T x_{t}\right\| \limsup _{n \rightarrow \infty}\left\|x_{t}-x_{n}\right\| .
\end{aligned}
$$

Taking the lim sup as $t \rightarrow 0$ and recalling (42) and the continuity of $F$, we conclude that

$$
\limsup _{t \rightarrow 0} \limsup _{n \rightarrow \infty}\left\langle(\mu F-\gamma V) x_{t}, j\left(x_{t}-x_{n}\right)\right\rangle \leq 0 .
$$

On the other hand, since $X$ is a real 2-uniformly smooth Banach space, and $J$ is single-valued and strong-weak ${ }^{*}$ uniformly continuous on $X$, as $t \rightarrow 0^{+}$, we have

$$
\begin{aligned}
\langle(\mu F & \left.-\gamma V) \tilde{x}, j_{q}\left(\tilde{x}-x_{n}\right)\right\rangle-\left\langle\mu F x_{t}-\gamma V x_{t}, j_{q}\left(x_{t}-x_{n}\right)\right\rangle \\
= & \left\langle(\mu F-\gamma V) \tilde{x}, j_{q}\left(\tilde{x}-x_{n}\right)-j_{q}\left(x_{t}-x_{n}\right)\right\rangle \\
& +\left\langle\mu F(\tilde{x})-\mu F x_{t}+\gamma V\left(x_{t}\right)-\gamma V(\tilde{x}), j_{q}\left(x_{t}-x_{n}\right)\right\rangle \\
= & \left\langle(\mu F-\gamma V) \tilde{x}, j_{q}\left(\tilde{x}-x_{n}\right)-j_{q}\left(x_{t}-x_{n}\right)\right\rangle \\
& +\left\langle\mu F(\tilde{x})-\mu F x_{t}, j_{q}\left(x_{t}-x_{n}\right)\right\rangle \\
& +\gamma\left\langle V\left(x_{t}\right)-V(\tilde{x}), j_{q}\left(x_{t}-x_{n}\right)\right\rangle \longrightarrow 0 .
\end{aligned}
$$

Thus, from (59) and (60), we obtain

$$
\begin{aligned}
& \limsup _{n \rightarrow \infty}\left\langle(\mu F-\gamma V) \tilde{x}, j_{q}\left(\tilde{x}-x_{n}\right)\right\rangle \\
& \quad=\limsup _{t \rightarrow 0} \limsup _{n \rightarrow \infty}\left\langle(\mu F-\gamma V) \tilde{x}, j_{q}\left(\tilde{x}-x_{n}\right)\right\rangle \leq 0 .
\end{aligned}
$$

So (55) is valid. This completes the proof.

Finally, we study the following implicit iterative method process: the initial $x_{0} \in X$ is arbitrarily selected, and the iterative algorithm is recursively defined by

$$
\begin{aligned}
x_{n}= & \alpha_{n} \gamma V\left(x_{n}\right)+\beta_{n} x_{n-1} \\
& +\left(\left(1-\beta_{n}\right) I-\mu \alpha_{n} F\right) T_{n} x_{n}, \quad \forall n \geq 1,
\end{aligned}
$$


where the sequences $\left\{\alpha_{n}\right\}$ and $\left\{\beta_{n}\right\}$ are sequences in $(0,1)$ and satisfy the following conditions:

(C1) $\lim _{n \rightarrow \infty} \alpha_{n}=\lim _{n \rightarrow \infty} \beta_{n}=0$,

(C2) $\sum_{n=0}^{\infty}\left(\alpha_{n} /\left(\alpha_{n}+\beta_{n}\right)\right)=\infty$.

Theorem 11. Let $\left\{T_{i}\right\}_{i=1}^{\infty}$ be a countable family of selfnonexpansive mappings on $X$, such that $S:=\bigcap_{n=1}^{\infty} \operatorname{Fix}\left(T_{n}\right) \neq \emptyset$. Let $V$ be an $L$-Lipschitzian continuous self-mapping on $X$ with Lipschitzian constant $L>0 . F: X \rightarrow X$ is an $\eta$-strongly accretive and $\kappa$-Lipschitzian continuous mapping with $\kappa>0$ and $\eta>0$. Suppose that the sequences $\left\{\alpha_{n}\right\}$ and $\left\{\beta_{n}\right\}$ satisfy the controlling conditions $(C 1)-(C 2)$. Let $\mu \in\left(0, \min \left\{1, \eta / K^{2} \kappa^{2}\right\}\right)$, and set $\tau=\mu\left(\eta-\mu K^{2} \kappa^{2}\right)$ and $(\tau-1) / L<\gamma<\tau / L$. Assume that $\left(\left\{T_{n}\right\}, T\right)$ satisfies the AKTT condition. Then $\left\{x_{n}\right\}$ defined by (62) converges strongly to a common fixed point $\tilde{x}$ of $\left\{T_{n}\right\}_{n=1}^{\infty}$ which equivalently solves the following variational inequality:

$$
\langle(\gamma V-\mu F) \tilde{x}, j(\tilde{x}-p)\rangle \geq 0, \quad \forall p \in S .
$$

Proof. First we show that $\left\{x_{n}\right\}$ is well defined. Consider a mapping $S_{n}$ on $X$ defined by

$$
S_{n} x=\alpha_{n} \gamma V x+\beta_{n} x_{n-1}+\left(\left(1-\beta_{n}\right) I-\mu \alpha_{n} F\right) T_{n} x .
$$

It is easy to see that $S_{n}$ is a contraction. Indeed, for any $x, y \in$ $X$, by Lemma 5 , we have

$$
\begin{aligned}
\| S_{t} x & -S_{t} y \| \\
\leq & \alpha_{n} \gamma\|V(x)-V(y)\| \\
\quad & +\left\|\left(\left(1-\beta_{n}\right) I-\alpha_{n} \mu F\right) T x-\left(\left(1-\beta_{n}\right) I-\alpha_{n} \mu F\right) T y\right\| \\
\leq & {\left[1-\beta_{n}-\alpha_{n}(\tau-\gamma L)\right]\|x-y\| . }
\end{aligned}
$$

Hence, $S_{n}$ is a contraction. By the Banach contraction mapping principle, we conclude that $S_{n}$ has a unique fixed point, denoted by $x_{n}$. So (62) is well defined.

Then we show that $\left\{x_{n}\right\}$ is bounded. Taking any $p \in S$, we have

$$
\begin{aligned}
\| x_{n}- & p \|^{2} \\
= & \alpha_{n}\left\langle\gamma V\left(x_{n}\right)-\mu F p, j\left(x_{n}-p\right)\right\rangle \\
& +\beta_{n}\left\langle x_{n-1}-p, j\left(x_{n}-p\right)\right\rangle \\
& +\left\langle\left(\left(1-\beta_{n}\right) I-\alpha_{n} \mu F\right) T_{n} x_{n}\right. \\
& \left.\quad-\left(\left(1-\beta_{n}\right) I-\alpha_{n} \mu F\right) p, j\left(x_{n}-p\right)\right\rangle \\
\leq & \alpha_{n} \gamma\left\langle V\left(x_{n}\right)-V(p), j\left(x_{n}-p\right)\right\rangle \\
+ & \alpha_{n}\left\langle\gamma V(p)-\mu F p, j\left(x_{n}-p\right)\right\rangle \\
+ & \beta_{n}\left\langle x_{n-1}-p, j\left(x_{n}-p\right)\right\rangle \\
+ & \left\langle\left(\left(1-\beta_{n}\right) I-\alpha_{n} \mu F\right) T_{n} x_{n}\right. \\
& \left.\quad-\left(\left(1-\beta_{n}\right) I-\alpha_{n} \mu F\right) p, j\left(x_{n}-p\right)\right\rangle
\end{aligned}
$$

$$
\begin{aligned}
\leq & \alpha_{n} \gamma L\left\|x_{n}-p\right\|^{2}+\alpha_{n}\left\langle\gamma V(p)-\mu F p, j\left(x_{n}-p\right)\right\rangle \\
& +\beta_{n}\left\|x_{n-1}-p\right\|\left\|x_{n}-p\right\| \\
& +\left(1-\beta_{n}-\alpha_{n} \tau\right)\left\|x_{n}-p\right\|^{2} \\
= & \left(1-\beta_{n}-\alpha_{n}(\tau-\gamma L)\right)\left\|x_{n}-p\right\|^{2} \\
& +\alpha_{n}\|\gamma V(p)-\mu F p\|\left\|x_{n}-p\right\| \\
& +\beta_{n}\left\|x_{n-1}-p\right\|\left\|x_{n}-p\right\|,
\end{aligned}
$$

which implies that

$$
\begin{aligned}
\left\|x_{n}-p\right\| \leq & \frac{\beta_{n}}{\beta_{n}+\alpha_{n}(\tau-\gamma L)}\left\|x_{n-1}-p\right\| \\
& +\frac{\alpha_{n}(\tau-\gamma L)}{\beta_{n}+\alpha_{n}(\tau-\gamma L)} \frac{\|\gamma V(p)-\mu F p\|}{\tau-\gamma L} .
\end{aligned}
$$

By induction, it follows that

$$
\left\|x_{n}-p\right\| \leq \max \left\{\left\|x_{0}-p\right\|, \frac{\|\gamma V(p)-\mu F p\|}{\tau-\gamma L}\right\}, \quad n \geq 0 .
$$

Hence $\left\{x_{n}\right\}$ is bounded, so are the $\left\{V\left(x_{n}\right)\right\}$ and $\left\{F\left(x_{n}\right)\right\}$.

Next, we show that

$$
\left\|x_{n}-T x_{n}\right\| \longrightarrow 0
$$

From (C1) and the definition of $\left\{x_{n}\right\}$, we observe that

$$
\begin{aligned}
&\left\|x_{n}-T_{n} x_{n}\right\| \\
&=\left\|\alpha_{n}\left(\gamma V\left(x_{n}\right)-\mu F T_{n} x_{n}\right)+\beta_{n}\left(x_{n-1}-T_{n} x_{n}\right)\right\| \\
& \leq \alpha_{n}\left\|\gamma V\left(x_{n}\right)-\mu F T_{n} x_{n}\right\| \\
&+\beta_{n}\left\|x_{n-1}-T_{n} x_{n}\right\| \longrightarrow 0 .
\end{aligned}
$$

By Lemma 7 and (70), we have

$$
\begin{aligned}
\left\|x_{n}-T x_{n}\right\|= & \left\|x_{n}-T_{n} x_{n}+T_{n} x_{n}-T x_{n}\right\| \\
\leq & \left\|x_{n}-T_{n} x_{n}\right\| \\
& +\left\|T_{n} x_{n}-T x_{n}\right\| \longrightarrow 0 .
\end{aligned}
$$

Let $x_{t}$ be defined by (33), from Propositions 9 and 10, and we have that $\left\{x_{t}\right\}$ converges strongly to $\widetilde{x} \in S:=\operatorname{Fix}(T)=$ $\bigcap_{i=1}^{\infty} \operatorname{Fix}\left(T_{i}\right)$ and

$$
\limsup _{n \rightarrow \infty}\left\langle(\gamma V-\mu F) \tilde{x}, j\left(x_{n}-\tilde{x}\right)\right\rangle \leq 0 .
$$


As required, finally we show that $x_{n} \rightarrow \tilde{x}$. As a matter of fact, by Lemmas 5 and 8 , we have

$$
\begin{aligned}
& \left\|x_{n}-\tilde{x}\right\|^{2} \\
& =\left\langle\left(\left(1-\beta_{n}\right) I-\alpha_{n} \mu F\right) T_{n} x_{n}-\left(\left(1-\beta_{n}\right) I-\alpha_{n} \mu F\right) \tilde{x}\right. \\
& \left.+\beta_{n}\left(x_{n-1}-\tilde{x}\right)+\alpha_{n}\left(\gamma V\left(x_{n}\right)-\mu F \tilde{x}\right), j\left(x_{n}-\tilde{x}\right)\right\rangle \\
& \leq\left\langle\left(\left(1-\beta_{n}\right) I-\alpha_{n} \mu F\right) T_{n} x_{n}\right. \\
& \left.-\left(\left(1-\beta_{n}\right) I-\alpha_{n} \mu F\right) \tilde{x}, j\left(x_{n}-\tilde{x}\right)\right\rangle \\
& +\beta_{n}\left\|x_{n-1}-\tilde{x}\right\|\left\|x_{n}-\tilde{x}\right\| \\
& +\alpha_{n}\left\langle\gamma V\left(x_{n}\right)-\mu F \tilde{x}, j\left(x_{n}-\tilde{x}\right)\right\rangle \\
& \leq\left(1-\beta_{n}-\alpha_{n} \tau\right)\left\|x_{n}-\tilde{x}\right\|^{2} \\
& +\beta_{n}\left\|x_{n-1}-\tilde{x}\right\|\left\|x_{n}-\tilde{x}\right\| \\
& +\alpha_{n} \gamma\left\langle V\left(x_{n}\right)-V(\tilde{x}), j\left(x_{n}-\tilde{x}\right)\right\rangle \\
& +\alpha_{n}\left\langle\gamma V(\tilde{x})-\mu F \tilde{x}, j\left(x_{n}-\tilde{x}\right)\right\rangle \\
& \leq\left(1-\beta_{n}-\alpha_{n} \tau\right)\left\|x_{n}-\tilde{x}\right\|^{2} \\
& +\beta_{n}\left\|x_{n-1}-\tilde{x}\right\|\left\|x_{n}-\tilde{x}\right\| \\
& +\alpha_{n} \gamma L\left\|x_{n}-\tilde{x}\right\|^{2}+\alpha_{n}\left\langle\gamma V(\tilde{x})-\mu F \tilde{x}, j\left(x_{n}-\tilde{x}\right)\right\rangle \\
& \leq\left(1-\beta_{n}-\alpha_{n}(\tau-\gamma L)\right)\left\|x_{n}-\tilde{x}\right\|^{2} \\
& +\beta_{n}\left\|x_{n-1}-\tilde{x}\right\|\left\|x_{n}-\tilde{x}\right\| \\
& +\alpha_{n}\left\langle\gamma V(\tilde{x})-\mu F \tilde{x}, j\left(x_{n}-\tilde{x}\right)\right\rangle \\
& \leq\left(1-\beta_{n}-\alpha_{n}(\tau-\gamma L)\right)\left\|x_{n}-\tilde{x}\right\|^{2} \\
& +\frac{\beta_{n}}{2}\left(\left\|x_{n-1}-\tilde{x}\right\|^{2}+\left\|x_{n}-\tilde{x}\right\|^{2}\right) \\
& +\alpha_{n}\left\langle\gamma V(\tilde{x})-\mu F \tilde{x}, j\left(x_{n}-\tilde{x}\right)\right\rangle \\
& \leq\left(1-\frac{\beta_{n}}{2}-\alpha_{n}(\tau-\gamma L)\right)\left\|x_{n}-\tilde{x}\right\|^{2} \\
& +\frac{\beta_{n}}{2}\left\|x_{n-1}-\tilde{x}\right\|^{2} \\
& +\alpha_{n}\left\langle\gamma V(\tilde{x})-\mu F \tilde{x}, j\left(x_{n}-\tilde{x}\right)\right\rangle,
\end{aligned}
$$

which implies that

$$
\begin{aligned}
\left\|x_{n}-\tilde{x}\right\|^{2} & \\
\leq & \frac{\beta_{n}}{\beta_{n}+2 \alpha_{n}(\tau-\gamma L)}\left\|x_{n-1}-\tilde{x}\right\|^{2} \\
& +\frac{2 \alpha_{n}}{\beta_{n}+2 \alpha_{n}(\tau-\gamma L)}\left\langle(\gamma V-\mu F) \tilde{x}, j\left(x_{n}-\tilde{x}\right)\right\rangle \\
= & {\left[1-\frac{2 \alpha_{n}(\tau-\gamma L)}{\beta_{n}+2 \alpha_{n}(\tau-\gamma L)}\right]\left\|x_{n-1}-\tilde{x}\right\|^{2} } \\
& +\frac{2 \alpha_{n}(\tau-\gamma L)}{\beta_{n}+2 \alpha_{n}(\tau-\gamma L)} \frac{\left\langle(\gamma V-\mu F) \tilde{x}, j\left(x_{n}-\tilde{x}\right)\right\rangle}{\tau-\gamma L} .
\end{aligned}
$$

It is easily to see that

$$
\frac{2 \alpha_{n}(\tau-\gamma L)}{\beta_{n}+2 \alpha_{n}(\tau-\gamma L)}>\frac{2 \alpha_{n}(\tau-\gamma L)}{2 \beta_{n}+2 \alpha_{n}}=(\tau-\gamma L) \frac{\alpha_{n}}{\alpha_{n}+\beta_{n}} .
$$

Thus, (C2) yields that $\sum_{n=0}^{\infty}\left(2 \alpha_{n}(\tau-\gamma L) /\left(\beta_{n}+2 \alpha_{n}(\tau-\gamma L)\right)\right)=$ $\infty$. Applying Lemma 4 and (72) to (74), we conclude that $x_{n} \rightarrow \tilde{x}$.

This completes the proof.

Remark 12. Our result in Proposition 9 extends Theorem 3.1 of Tian [8] from real Hilbert spaces to real 2-uniformly smooth Banach spaces. If we set $\beta_{n}=0$, our result in Theorem 11 extends Theorem 3.2 of Tian [8] from real Hilbert spaces to real 2-uniformly smooth Banach spaces as well as from a single nonexpansive mapping to a countable family of nonexpansive mappings.

Remark 13. In 2008, Hu [14] introduced a modified Halperntype iteration for a single nonexpansive mapping in Banach spaces which have a uniformly Gêteaux differentiable norm as follows:

$$
x_{n+1}=\alpha_{n} u+\beta_{n} x_{n}+\gamma_{n} T x_{n} .
$$

Under some appropriate assumptions, he proved that the sequence $\left\{x_{n}\right\}$ defined by the iteration process (76) converges strongly to the fixed point of $T$.

Corollary 14. If we take $\gamma=1, F=I, \mu=1$, and $\beta_{n}=0$ in (62), we extend the classical viscosity approximation [4] under a mild assumption: the contraction mapping $f$ is replaced by an L-Lipschitzian continuous mapping V. Our proving process needs no Banach limit and is different from the proving process given by $\mathrm{Xu}[6]$ in some aspects.

Remark 15. Ceng et al. [11] introduced the following iterative algorithm to find a common fixed point of a finite family of nonexpansive semigroups in reflexive Banach spaces:

$$
\begin{gathered}
x_{n+1}=\left(1-\alpha_{n}-\beta_{n}\right) x_{n}+\alpha_{n} f\left(y_{n}\right)+\beta_{n} T_{r_{n}} y_{n}, \\
y_{n}=\left(1-\gamma_{n}\right) x_{n}+\gamma_{n} T_{r_{n}} x_{n} .
\end{gathered}
$$

Under some appropriate conditions one the parameter sequences $\left\{\alpha_{n}\right\},\left\{\beta_{n}\right\},\left\{\gamma_{n}\right\}$, and the sequence $\left\{x_{n}\right\}$ converges strongly to the approximate solution of a variational inequality problem.

If we set $\alpha_{n}+\beta_{n}=1$ and $\gamma_{n}=0$, the algorithm is simplified into viscosity-form iterative schemes for a finite family of nonexpansive semigroups. Our algorithms are considered in full space and avoid the generalized projections or sunny nonexpansive retractions in Banach space. For further improving our works, in order to obtain more general results, we should take the results given by Ceng et al. in [11] into account.

\section{Acknowledgments}

Ming Tian was supported in part by the Fundamental Research Funds for the Central Universities (no. ZXH2012K001). Xin Jin was supported in part by Technology Innovation Funds of Civil Aviation University of China for Graduate (YJSCX12-18). 


\section{References}

[1] D. S. Mitrinović, Analysis Inequality, Springer, New York, NY, USA, 1970.

[2] M.-M. Wong, Q. H. Ansari, and J.-C. Yao, "Existence of solutions of generalized variational inequalities in reflexive Banach spaces," Applied Mathematics Letters of Rapid Publication, vol. 22, no. 2, pp. 197-201, 2009.

[3] I. Yamada, "The hybrid steepest descent method for the variational inequality problem over the intersection of fixed point sets of nonexpansive mappings," in Inherently Parallel Algorithms in Feasibility and Optimization and Their Applications, S. H. D. Butnariu, Y. Censor, and S. Reich, Eds., vol. 8, pp. 473504, North-Holland, Amsterdam, The Netherlands, 2001.

[4] A. Moudafi, "Viscosity approximation methods for fixed-points problems," Journal of Mathematical Analysis and Applications, vol. 241, no. 1, pp. 46-55, 2000.

[5] H. K. Xu, "An iterative approach to quadratic optimization," Journal of Optimization Theory and Applications, vol. 116, no. 3, pp. 659-678, 2003.

[6] H.-K. Xu, "Viscosity approximation methods for nonexpansive mappings," Journal of Mathematical Analysis and Applications, vol. 298, no. 1, pp. 279-291, 2004.

[7] G. Marino and H.-K. Xu, "A general iterative method for nonexpansive mappings in Hilbert spaces," Journal of Mathematical Analysis and Applications, vol. 318, no. 1, pp. 43-52, 2006.

[8] M. Tian, "A general iterative algorithm for nonexpansive mappings in Hilbert spaces," Nonlinear Analysis. Theory, Methods \& Applications A, vol. 73, no. 3, pp. 689-694, 2010.

[9] S. M. Soltuz, “The backward Mann iteration," Octogon Mathematical Magazine, vol. 9, pp. 797-800, 2001.

[10] H.-K. Xu and R. G. Ori, "An implicit iteration process for nonexpansive mappings," Numerical Functional Analysis and Optimization, vol. 22, no. 5-6, pp. 767-773, 2001.

[11] L.-C. Ceng, Q. H. Ansari, and J. C. Yao, "On relaxed viscosity iterative methods for variational inequalities in Banach spaces," Journal of Computational and Applied Mathematics, vol. 230, no. 2, pp. 813-822, 2009.

[12] L.-C. Ceng, Q. H. Ansari, and J.-C. Yao, "Relaxed extragradient iterative methods for variational inequalities," Applied Mathematics and Computation, vol. 218, no. 3, pp. 1112-1123, 2011.

[13] Y. Yao, Y.-C. Liou, and R. Chen, "Strong convergence of an iterative algorithm for pseudocontractive mapping in Banach spaces," Nonlinear Analysis. Theory, Methods \& Applications A, vol. 67, no. 12, pp. 3311-3317, 2007.

[14] L.-G. Hu, “Strong convergence of a modified Halpern's iteration for nonexpansive mappings," Fixed Point Theory and Applications, vol. 2008, Article ID 649162, 9 pages, 2008.

[15] J. S. Jung, "Some results on a general iterative method for $k$ strictly pseudo-contractive mappings," Fixed Point Theory and Applications, vol. 2011, 11 pages, 2011.

[16] D. F. Cudia, "The geometry of Banach spaces. Smoothness," Transactions of the American Mathematical Society, vol. 110, pp. 284-314, 1964.

[17] H. K. Xu, "Inequality in Banach spaces with applications," Nonlinear Analysis, vol. 16, pp. 1127-1138, 1991.

[18] T.-C. Lim and H. K. Xu, "Fixed point theorems for asymptotically nonexpansive mappings," Nonlinear Analysis. Theory, Methods \& Applications A, vol. 22, no. 11, pp. 1345-1355, 1994.

[19] H. K. Xu, "Iterative algorithm for nonlinear operator," Journal of the London Mathematical Society, vol. 66, pp. 240-256, 2002.
[20] K. Aoyama, Y. Kimura, and W. Takahashi, "Strong convergence of common fixed points of a countable family of nonexpansive mappings in Banach space," Nonlinear Analysis. Theory, Methods \& Applications A, vol. 67, pp. 2350-2360, 2007. 


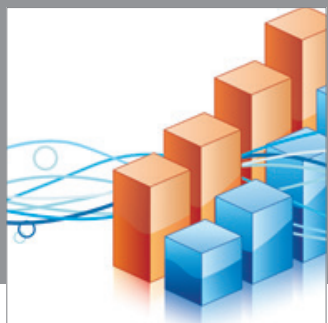

Advances in

Operations Research

mansans

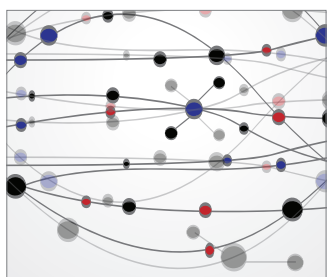

The Scientific World Journal
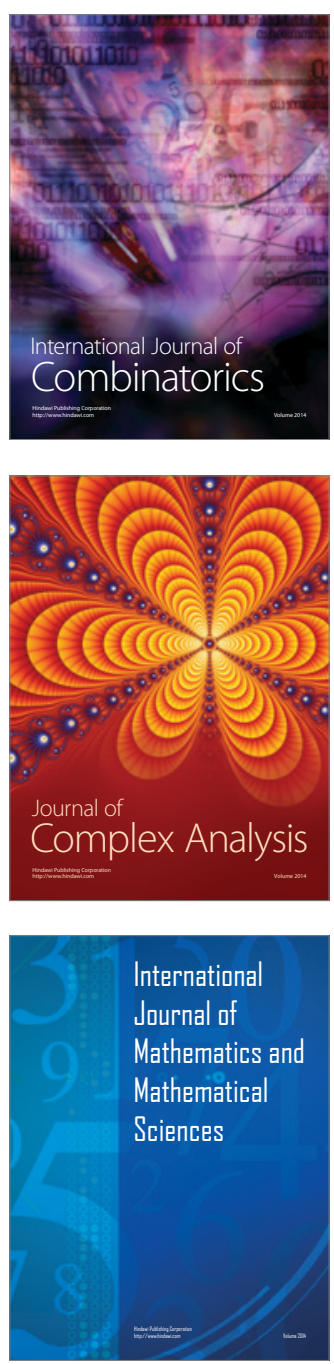
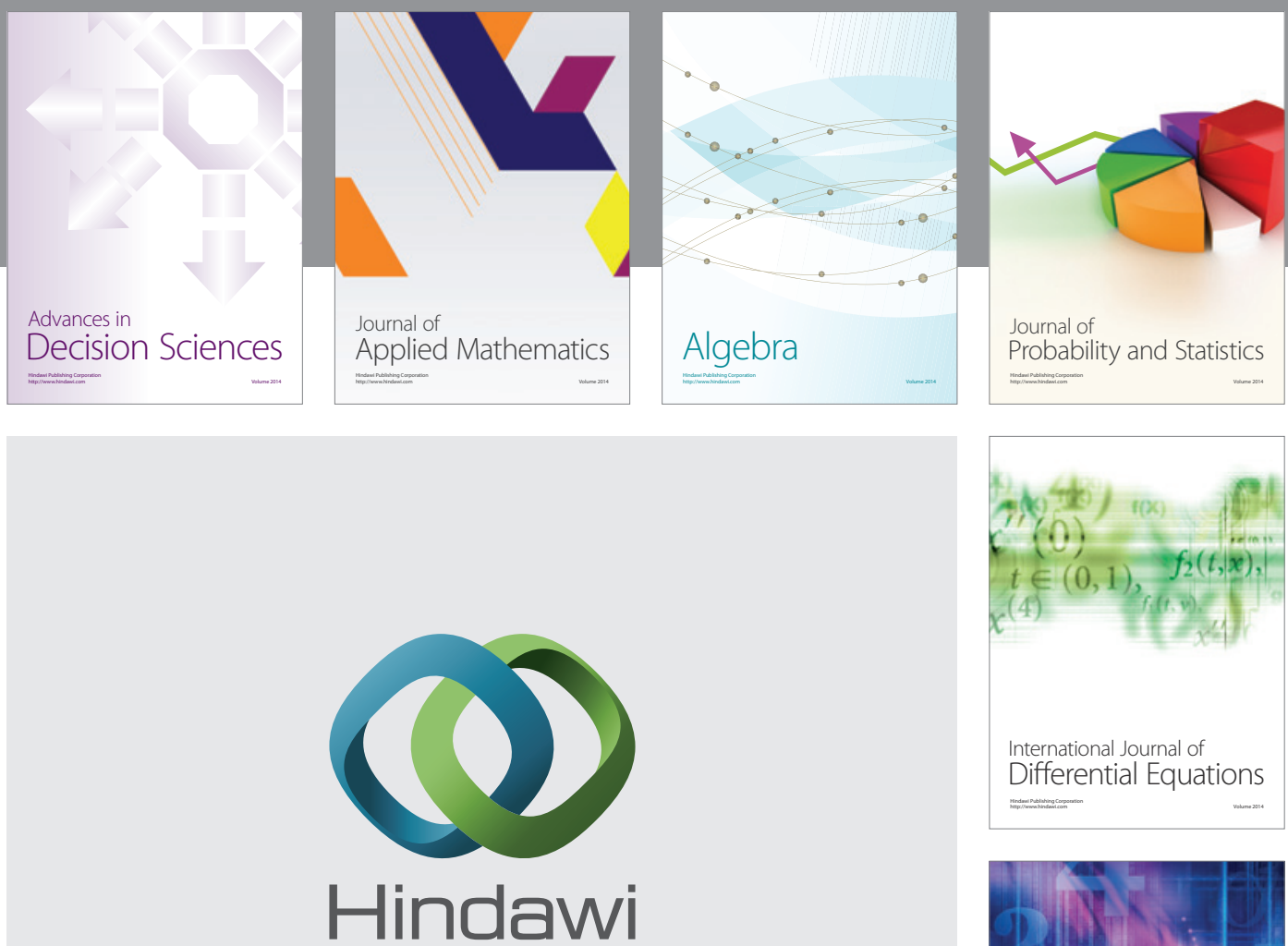

Submit your manuscripts at http://www.hindawi.com
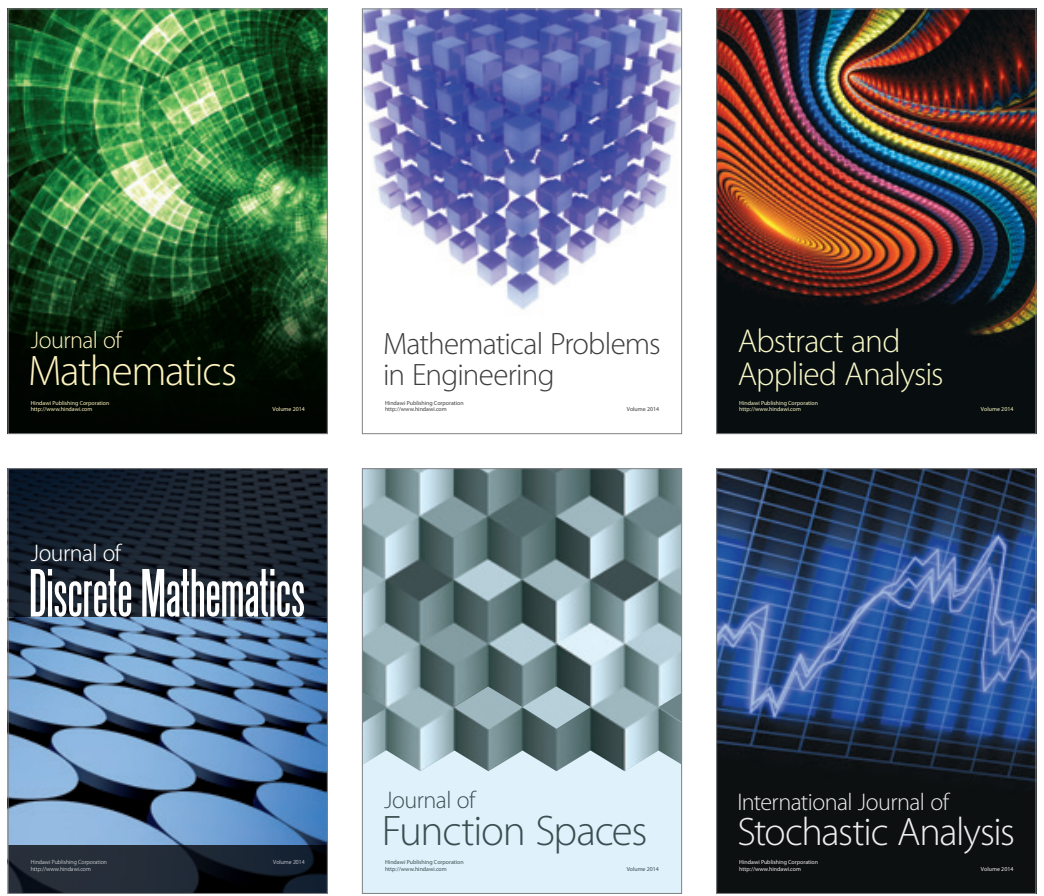

Journal of

Function Spaces

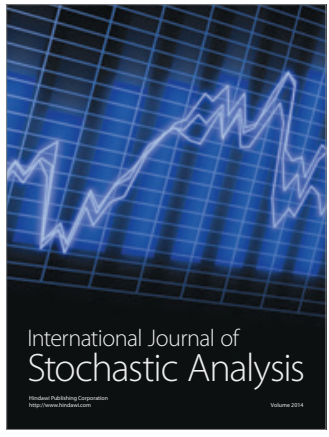

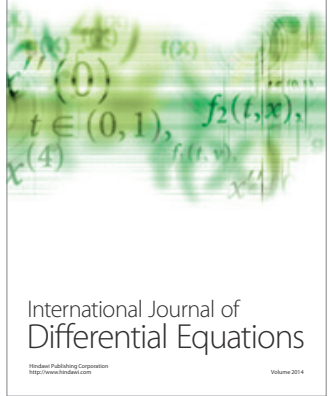
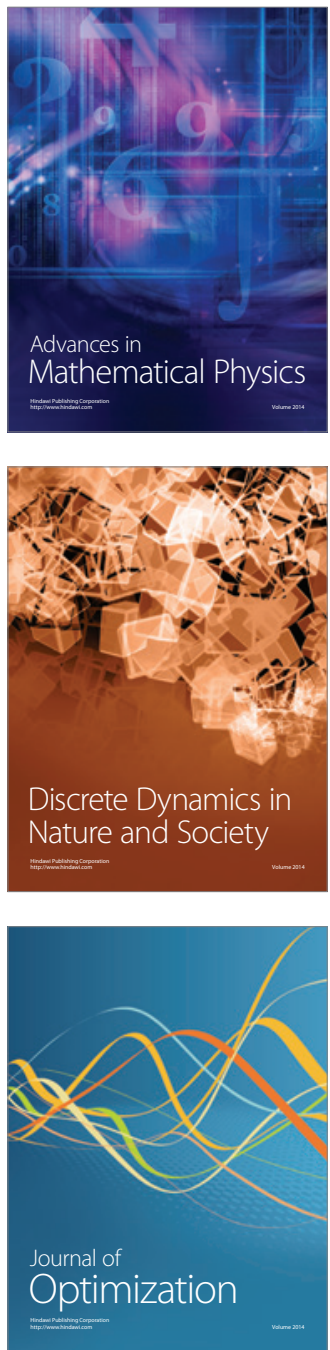